\title{
A humanização da merenda escolar na promoção da saúde e da educação pública: uma breve revisão
}

\author{
The humanization of school lunches in the promotion of health and public education: a \\ brief review
}

La humanización de los almuerzos escolares en la promoción de la salud y la educación pública: una breve reseña

Lázaro Saluci Ramos $^{1 *}$, Eliane Maria Ferreira Moreira ${ }^{2}$, Fernanda Aparecida da Silva ${ }^{3}$, Elza Aline Moura Nazario ${ }^{3}$, Leonardo Ribeiro Pereira Sobrinho ${ }^{3}$, Derlucia do Nascimento Brito ${ }^{3}$, Kadjas Púperi Monteiro ${ }^{3}$, Ozéas Teixeira Neves ${ }^{3}$, Rafael Perin dos Santos ${ }^{3}$, Edivaldo Menegazzo de Almeida ${ }^{3}$.

\section{RESUMO}

Objetivo: Objetiva-se no presente artigo apontar os benefícios de uma merenda escolar humanizada à fim de aumentar o coeficiente educacional e ampliar os cuidados com a saúde dos adolescentes. Revisão Bibliográfica: Há que se considerar que a saúde alimentar influi muito no aprendizado e nas condições mínimas para que um aluno possa desenvolver o intelecto e garantir o aprendizado. A humanização da merenda escolar gira em torno de oferecer alimentos nobres a saúde dos alunos a fim de garantir a promoção educacional e reduzir a incidência de doenças causadas pela má alimentação. Produtos orgânicos, rigorosamente balanceados por profissionais da nutrição além de educação alimentar, serão portas solucionadoras de problemas na educação pública além de construir uma alternativa para ampliar o aumento de recursos em outras áreas da saúde pública. É possível de igual forma, construir em conjunto ao ambiente escolar, políticas informativas para estender aos lares dos alunos a continuidade da alimentação humanizada. Considerações finais: Conclui-se, portanto, que garantir uma alimentação humanizada no ambiente escolar soluciona problemas de duas áreas amplamente afetadas por falta de inovações no setor público brasileiro.

Palavras-chave: Educação alimentar e nutricional, Nutrição, Alimentação escolar.

\begin{abstract}
Objective: The objective of this article is to point out the benefits of a humanized school lunch in order to increase the educational coefficient and expand the health care of adolescents. Bibliographic Review: It is necessary to consider that food health has a great influence on learning and on the minimum conditions for a student to develop his intellect and guarantee learning. The humanization of school lunches revolves around offering noble foods to students' health in order to guarantee educational promotion and reduce the incidence of diseases caused by poor diet. Organic products, strictly balanced by nutrition professionals in addition to food education, will be problem solving doors in public education as well as building an alternative to increase the increase in resources in other areas of public health. It is also possible, together with the school environment, to build informative policies to extend the continuity of humanized feeding to students' homes. Final considerations: It is concluded, therefore, that guaranteeing humanized food in the school environment solves problems in two areas that are largely affected by the lack of innovations in the Brazilian public sector.
\end{abstract}

Key words: Food and nutrition education, Nutrition, School feeding.

\footnotetext{
${ }^{1}$ Faculdade Multivix (MULTIVIX), Cachoeiro de Itapemirim - ES. *E-mail: lazaro321123@gmail.com

2 Universidade Unigranrio (UNIGRANRIO), Rio de Janeiro - RJ.

${ }^{3}$ Faculdade Vale do Cricaré (FVC), São Mateus - ES.
} 


\section{RESUMEN}

Objetivo: El objetivo de este artículo es señalar los beneficios de un almuerzo escolar humanizado con el fin de aumentar el coeficiente educativo y ampliar la atención médica de los adolescentes. Revisión bibliográfica: es necesario tener en cuenta que la salud alimentaria tiene una gran influencia en el aprendizaje y en las condiciones mínimas para que un estudiante desarrolle su intelecto y garantice el aprendizaje. La humanización de los almuerzos escolares gira en torno a ofrecer alimentos nobles a la salud de los estudiantes para garantizar la promoción educativa y reducir la incidencia de enfermedades causadas por una dieta deficiente. Los productos orgánicos, estrictamente equilibrados por los profesionales de la nutrición, además de la educación alimentaria, serán puertas para resolver problemas en la educación pública, así como construir una alternativa para aumentar el aumento de recursos en otras áreas de la salud pública. También es posible, junto con el entorno escolar, desarrollar políticas informativas para extender la continuidad de la alimentación humanizada a los hogares de los estudiantes. Consideraciones finales: por lo tanto, se concluye que garantizar alimentos humanizados en el entorno escolar resuelve problemas en dos áreas que se ven afectadas en gran medida por la falta de innovaciones en el sector público brasileño.

Palabras clave: Educación alimentaria y nutricional, Nutrición, Alimentación escolar.

\section{INTRODUÇÃO}

A terminologia "merenda escolar" compreende todas as alimentações realizadas pelos alunos na escola durante o dia letivo. É de suma importância garantir a mínima alimentação diária de crianças e adolescentes, principalmente em regiões mais pobres, onde costumeiramente se encontra famílias que não fazem sequer três refeições ao dia. De tal importância se configura a alimentação no ambiente escolar, que se estrutura um plano de identidade do aluno com a alimentação saudável, garantindo saúde e consequentemente melhores condições de aprendizado (SILVA EO, et al., 2018).

Muitas regiões brasileiras adotam o sistema de ensino em tempo integral à fim de garantir melhor desenvolvimento intelectual e ao menos três refeições diárias ao aluno, principalmente em localizações mais pobres. Esse sistema frutificou a democratização do ensino e estendeu-a para a democratização do direito básico a alimentação. Foi uma forma que o Estado encontrou de atrair a criança e o adolescente ao ambiente escolar, e seguidamente de combater a desnutrição infantil e os constantes casos de doenças causadas pela má alimentação (SILVA EO, et al., 2018).

A influência do ambiente escolar na vida do aluno, não perpassa apenas pela área acadêmica, mas também pelos variados ambientes e recreações promovidas ao entorno. Incluir alimentação saudável no ambiente escolar é incentivar hábitos alimentares futuros mais adequados, é constituir incentivo e ampliar o foco do aluno na importância de se alimentar bem. Esse canal aberto entre escola e aluno, também servirá de entrada para inserir novas temáticas dentro de disciplinas que compreendem a área da saúde. Devolver o aluno para sociedade com consciência alimentar é um meio eficaz de construir uma sociedade mais saudável e mais disposta ao academicismo (MANCUSO AMC, et al., 2013).

A luz da didática nutricional expõe a possibilidade de inserir na educação básica brasileira, a princípio dentro das disciplinas já existentes e posteriormente, com a ampliação das escolas de educação em tempo integral, através da criação de uma nova disciplina específica, a educação alimentar básica. Aulas de gastronomia, ministradas por especialistas e profissionais da área da nutrição, e com uma grade ampla abrangendo não só os benefícios de uma alimentação saudável para a saúde, mas a importância do consumo e do cultivo de alimentos orgânicos, para humanizar a alimentação da população (MANCUSO AMC, et al., 2016).

O Programa Nacional de Alimentação Escolar (PNAE), prioriza alimentos orgânicos, sem agrotóxicos ou qualquer tipo de produto químico nocivo à saúde humana, para a alimentação escolar. Com base nessa abordagem, ações no ambiente escolar voltadas a educação sobre o cultivo de alimentos, como a confecção de hortas, estufas e laboratórios que auxiliam na seleção dos grãos, por exemplo, são preferenciais e 
amplamente incentivadas. $O$ apoio das disciplinas das ciências da saúde é de total importância para o amadurecimento e a constância dessas atividades fora do ambiente escolar. As escolas técnicas agrícolas, Institutos Federais (IF) de educação, dentre outras instituições que promovem a educação no campo ou para o campo, são notórios exemplos de apoio ao desenvolvimento científico e incentivo a pesquisa. (CUNHA E, et al., 2010).

Esta revisão tem como objetivo a inserção de políticas de saúde pública no ambiente escolar a fim de garantir maior eficácia na construção do ensino, na alimentação saudável e humanizada, e no combate a doenças causadas pela má alimentação ou pela contaminação por agrotóxicos. Surge também como uma nova proposta a educação básica brasileira, políticas de saúde alimentar dentro da grade escolar, como garantia de que o aluno exportará o conhecimento adquirido para fora da escola, incluindo toda sociedade em uma grande reforma alimentar.

\section{REVISÃO BIBLIOGRÁFICA}

Humanizar está intimamente ligado a tornar humano, dar dignidade ao ser humano e garantir-Ihe tratamento digno. A intenção em buscar uma merenda escolar humanizada é garantir aos alunos não somente o mínimo de refeições diárias, mas uma expectativa de vida mais longeva, com qualidade e imunidade a doenças. A estrutura escolar veio sofrendo mudanças na última década, mas ainda carece de colaboração, recursos e fiscalização para tornar possível uma qualidade eficiente na comida servida nas escolas (LIMA EE e SOUSA AA, 2011).

Inúmeras mudanças nos hábitos alimentares recentemente foram conquistadas através do desenvolvimento tecnológico e pela globalização da informação. Tanto a Organização das Nações Unidas (ONU) como programas governamentais, estão inclinados resistentemente na construção de uma sociedade que se alimenta melhor. Atividades elaboradas para o ambiente escolar na intenção de promover saúde alimentar, estão intimamente ligadas a busca por uma saúde de qualidade, autoestima renovada nos alunos e habilidades bem desenvolvidas (CAMOZZI ABQ, et al., 2015).

Há através do PNAE o incentivo constante ao envolvimento da comunidade escolar no processo de humanização da merenda escolar e a promoção da educação alimentar e nutricional no processo de ensinoaprendizagem. Considera-se também que a atividade alimentar para o ser humano não acontece apoiada nas necessidades do corpo, somente, mas é influenciada por construções culturais e sociais, por isso a necessidade do amparo científico de profissionais da saúde, na reconstrução de hábitos que visam preservar ainda mais a vida biológica e a qualidade dela (CAMOZZI ABQ, et al., 2015).

A alimentação adequada é objeto da política mundial dos direitos humanos, sendo assim garantir ao ser humano condições mínimas de sobrevivência ainda é um desafio para sociedades contemporâneas, ainda soa distante os planos alimentares organizados e condizentes com as necessidades das pessoas. A produção de alimentos no planeta serve mais ao mercado que as reais necessidades do ser humano. Áreas completamente desmatadas de florestas servem ao agronegócio, mas o extrativismo sustentável que seria atividade mais equilibrada a saúde humana e do planeta, não arrenda lucros em grande escala (TEO CRPA, et al., 2017).

Dos diversos problemas que uma alimentação inadequada causa a crianças e adolescentes, um dos maiores deles, senão o maior, é a obesidade infantil. A obesidade infantil é o primeiro sinal de que na vida adulta haverá problemas com o controle do peso. A obesidade é uma das principais causadoras de hipertensão arterial e infartos no Brasil, além dos inúmeros danos que o excesso de gordura pode causar ao corpo. Problemas respiratórios, estomacais, redução da mobilidade e inclusive a depressão. O controle deve começar desde cedo, e o ambiente escolar é o melhor canal para introduzir políticas de saúde pública nesse sentido (BATISTA MSA, et al., 2014). É um direito humano garantido pela Declaração Universal dos Direitos Humanos, a alimentação adequada. Está disposto no artigo 25 da carta de 1948. Também foi restaurado esse direito em inúmeros pactos internacionais firmados por inúmeros Países. O Estado brasileiro busca promover a alimentação adequada através de programas alimentares, incentivo a agricultura familiar, garantia de 
alimentação nos ambientes escolares e principalmente promovendo políticas de geração de emprego, saúde, cultura e segurança (BRASIL, 2014).

\section{0 incentivo à alimentação orgânica}

O consumo de alimentos orgânicos no planeta aumenta consideravelmente a cada dia, principalmente após a divulgação de dados médicos sobre a incidência dos agrotóxicos como um dos principais causadores de câncer. No Brasil, os maiores produtores de alimentos orgânicos são os assentados do Movimento Sem Terra (MST) ou agricultores beneficiados pelos programas de financiamento a agricultura familiar e ao pequeno produtor rural. Outro benefício claro a saúde da população, geração de renda aos pequenos produtores rurais, combate à desigualdade social e aumento do poderio de consumo (LIMA EE e SOUSA AA, 2011).

O estado de São Paulo, promulgou a lei municipal 16.140 de março de 2015 que dispõe sobra a obrigatoriedade de inclusão de alimentos orgânicos ou de base agroecológica na alimentação escolar no âmbito do sistema municipal de ensino da cidade. Contudo, no ano de 2017 foi atestado que apenas 0,7\% da verba destinada ao programa foi utilizada, fazendo com que o programa se desgastasse e deixasse de fazer parte da política alimentar do estado (ALIMENTAÇÃO SAUDÁVEL, 2018).

"O projeto de Lei dizia o seguinte: Art. 2o Fica instituída a obrigatoriedade de inclusão de alimentos orgânicos ou de base agroecológica prioritariamente da agricultura familiar e do empreendedor familiar rural ou suas organizações, nos termos da Lei Federal no 11.326/2006, na alimentação escolar no âmbito do Sistema Municipal de Ensino. Art. 3 Entende-se por alimento orgânico ou de base agroecológica aquele produzido nos termos da Lei Federal no 10.831, de 23 de dezembro de 2003, ou a norma que vier a substituí-la, devidamente certificado ou produzido por agricultores familiares, que façam parte de uma Organização de Controle Social - OCS, cadastrada no Ministério da Agricultura, Pecuária e Abastecimento - MAPA, e tenham sido inscritos no Cadastro Nacional de Produtores Orgânicos ou em outro que venha a ser instituído no âmbito federal" (SÃO PAULO, 2015).

A garantia de que novas políticas nesse sentido vão se tornar realidade nas escolas do ensino básico brasileiro requer políticas públicas do Estado voltadas a inserir no cotidiano do aluno a preocupação com a boa alimentação, além disso tornar o ambiente escolar uma extensão das casas de saúde pública, afim de interagir a importância dos dois setores e ampliar o número de atores no desenvolvimento desse projeto. Quanto mais trouxer a sociedade civil para as proximidades do ambiente escolar, maiores as chances da política educacional alimentar obter resultados positivos (SANTOS F, et al., 2014). A garantia que se tem de que $\mathrm{o}$ alimento é orgânico é conseguida a partir de 3 formas diferentes: Certificado de auditores, que adota critérios de políticas internacionais; Sistemas Participativos de Garantia (SPG), formado por um Organismo Participativo de Avaliação da Conformidade (OPAC); Controle Social para a Venda Direta, no qual não se exige alguma certificação, funciona através da agricultura familiar em um cadastro organizacional de órgão de fiscalização municipal, estadual ou federal, garantindo, de tal forma, a comercialização direta ao comprador ou para programas de aquisição de alimentos da agricultura familiar (SANTOS F, et al., 2014).

Através das novas diretrizes do PNAE que vieram em 2009, passou-se a dar preferência a aquisição de alimentos da agricultura familiar para as escolas. A merenda orgânica e equilibrada se tornou objetivo indispensável, após se tornar objeto de programa federal, e contribuir para aumentar a renda de agricultores da agricultura familiar. Esse apoio do governo federal, não somente estimulou a produção de alimentos orgânicos, como valorizou a saúde dos próprios produtores, que passaram a não ter tanto contato com agrotóxicos (SILVA APF e SOUSA AA, 2013).

Para Coelho DEP e Bógus CM (2016); uma saída não menos acessível para tonar a merenda escolar orgânica e saudável é a implantação de hortas nas escolas. Além de colaborar com o ensino da prática do plantio e da autossutentabilidade, estabelece um meio de inserir noções mínimas de confiabilidade nos 
alunos, para a obtenção de alimentos que não estejam contaminados ou envenenados. Perpassa por uma questão extremamente pessoal e cultural o desenvolvimento de hábitos alimentares, entretanto, se torna um problema social grave quando a população deixa de estar atenta a quantidade de riscos que ela se expõe e a quantidade de vítimas alçadas aos leitos dos hospitais.

Muitas escolas adotaram o sistema de hortas, não somente para garantir a organicidade dos alimentos, mas também para driblar os problemas relacionados a ausência de recursos para a merenda. Nem sempre a merenda escolar foi balanceada e os recursos para adquirir alimentos foi suficiente. Em tempos pretéritos, principalmente nas regiões mais pobres do País, era muito comum até mesmo a venda de hortaliças para obtenção de recursos que seriam utilizados posteriormente na ampliação da estrutura do prédio escolar. Isso incentivou uma participação mais ativa da comunidade, e colaborou no incentivo a consciência alimentar (COELHO DEP e BOÓGUS CM, 2016).

\section{Educação alimentar na sala de aula}

Há tempos que os conceitos de escola variam constantemente. Não se diz mais que o ambiente escolar é para aprender apenas disciplinas de matemática, português, história e geografia, mas para ter noções básicas daquilo que compreende necessidades para a vida humana. Estabelecer novos padrões de ensino com grades que contemplem matérias de saúde básica, noções básicas de comportamento social entre outras adaptações necessárias para se inserir no meio social contemporâneo, é um desafio formidável aos pensadores da educação e aos profissionais das áreas afins (BARBOSA NVS, et al., 2013).

A inserção da educação alimentar nas escolas brasileiras consiste em aproveitar o espaço das disciplinas compreendidas pelas ciências da natureza para instruir o corpo discente acerca da importância de se comer bem, como adquirir alimento seguro, como cultivar alimento orgânico e quais os benefícios para a saúde das práticas renovadoras da alimentação saudável. Estudos apontam que um grande número dos problemas de saúde é causado na infância devido a alimentação irregular. A ausência de informação nutricional é um dos agravantes, já que a maior parte das famílias não conhecem os reais riscos que da obesidade infantil, e menos ainda táticas de policiar ou melhorar a alimentação das crianças (BEZERRA MA, et al., 2017).

Poucos jovens e adolescentes se interessam por assuntos que envolvem alimentação saudável e alimentos orgânicos, poucos deles sequer sabem do que se trata quando a palavra agrotóxico é mencionada. Muitas das vezes, comerciantes de agrotóxicos alteram o nome para defensivos agrícolas, e isso não é informado com clareza, mesmo a substância sendo nociva à saúde. A construção de um ambiente escolar informativo, com campanhas que visem alertar os alunos e através deles divulgar para a sociedade civil os riscos quanto a não observação da qualidade dos produtos, servirá como ponte para alcançar o objetivo central de extinguir o mercado de produtos contaminados (SOUSA AA, et al., 2012).

As disciplinas de Ciências e Biologia, que estão diretamente ligadas a saúde pública e trazem na grade noções básicas de saúde, podem abordar pontos importantes para a distribuição do conhecimento sobre alimentação segura e saudável. O ideal seria um profissional específico da nutrição, mas não se trata apenas de boa vontade, como também é necessário um massivo investimento de verba pública através de um projeto pré-analisado. A maneira mais rápida de garantir conhecimento mínimo acerca do assunto é utilizando as ferramentas que se tem, e para palear necessita-se de ideias implementáveis a curto prazo (SOUSA AA, et al., 2012).

A saúde na escola é um desafio e tanto para as futuras gerações, mas cabe as atuais dar o primeiro passo. A população só poderá ser mais autônoma, quando o assunto se tratar de saúde pública, a partir do momento que conhecimentos básicos de saúde pública forem para todos. Necessário se faz adotar mecanismos de ampla divulgação. O ideal se passa por utilizar estruturas já existentes na busca de acelerar o processo de implementação, e garantir que o primeiro passo não dependa de construções teóricas, mas também se passe pelo campo experimental prático (CASEMIRO JP, et al., 2014). A estrutura procurada já existente é a estrutura escolar, onde o conhecimento científico começar a se desenvolver e a instrução social pode ser aplicada, para solucionar problemas sociais e produzir resolução de conflitos históricos e culturais. A educação se tornou o maior canal de construção de políticas públicas da história da humanidade, pois é a partir da escola 
que se identificam os primeiros devaneios e problemas a se cuidar. E buscando solucionar esses devaneios, é que se pensa soluções práticas e complexas (CASEMIRO JP, et al., 2014).

Um projeto de Lei do ano de 2009 previa o profissional nutricionista na escola, mas foi rejeitado nas comissões de mérito. Com muito custo se conquistou o direito de profissionais da psicologia e da assistência social, o próximo passo certamente será buscar um ambiente escolar mais consciente no que toca a saúde alimentar. A sensibilidade dos parlamentares ainda não foi atingida pela necessidade de combater os problemas causados pela má alimentação. Muito mais se gasta com saúde pública no futuro, do que se gastaria atualmente implementando políticas preventivas (O GLOBO, 2018).

O projeto apontava que: Art. $1^{\circ}$ Obriga as escolas, públicas, de ensino fundamental e médio que distribuam merenda escolar aos alunos, a contratar pelo menos um nutricionista para o controle geral dos alimentos consumidos. Art. $2^{\circ}$ Os serviços de vigilância sanitária serão responsáveis pela fiscalização da qualidade dos alimentos distribuídos nas escolas e pela observância da presente lei (BRASIL, 2009).

A justificativa narra que atualmente cerca de 37 milhões de refeições são distribuídas diariamente para alunos de escolas de todo País, mas que não há nenhuma garantia de que esse alimento possua qualidade. Tocante a isto, relacionado a fragilidade da fiscalização e fazendo menção a pouca qualidade do que se oferta na escola, o projeto foi proposto. A justificativa também apontou estudos e pesquisadores que apontam a importância da qualidade da merenda escolar (BRASIL, 2009).

O conhecimento mínimo e básico sobre alimentação saudável nas escolas é uma medida preventiva, que busca solucionar velhos problemas e alçar novas propostas para o campo acadêmico. Quantas descobertas científicas não se pode alcançar aguçando o conhecimento sobre alimentação? Quantos novos cientistas para desenvolver pesquisas da área da saúde não serão integrados aos laboratórios das universidades e dos hospitais? Novas ideias surgem de novos integrantes, seres que respiram ares diferentes e focam em pontos complexos que nunca foram desvendados. Esse sentimento que pode e deve ser despertado nos alunos (FREITAS MCS, et al., 2013). É da natureza do escolar ter dúvidas, e quanto mais incentivo recebe em determinadas temáticas, mais dúvidas aparecem e constante a isso a vontade de desvendar. Um dos sérios problemas da educação brasileira gira em torno do pouco incentivo a novas aptidões e ao robotizado modo de transmissão de conhecimento. Muito pouco se propõe de novas técnicas para fortalecer a didática e democratizar o ensino, assim, fica uma eterna disputa entre os alunos, uma competição em busca de notas, avaliação suficiente para alcançar aprovação e nunca para adquirir mais conhecimento (DAVID CM, et al., 2015).

\section{CONSIDERAÇÕES FINAIS}

Conclui-se, portanto, que a busca incessante em inserir a saúde alimentar no âmbito da educação pública, é um desafio provador que ainda é tratado com descaso e pouca discussão. Tudo que se fez em nome da saúde alimentar dos alunos da rede pública de ensino, se for traçado desde a década de cinquenta, os ganhos foram exorbitantes, mas ainda assim é necessário investir em qualidade, unir esforços de ambos os setores é a principal ferramenta para a conquista desse feito. Instruir as futuras gerações quanto a importância de se alimentar bem, de preservar a saúde e buscar sempre o alimento mais saudável, é exportar conhecimento científico, crítico e promissor para a sociedade. $O$ aluno tem a missão, mesmo que não sabida, de distribuir o conhecimento adquirido na escola por onde passar. Ele o faz de forma natural, precisa apenas ser instruído da maneira correta. Os rumos das ciências estão debruçados na capacidade de ambas se ajudarem na construção de soluções.

\section{REFERÊNCIAS}

1. ALIMENTAÇÃO SAUDÁVEL. 2018. In: Lei que prevê merenda orgânica não atinge meta em São Paulo. São Paulo: Aliança Pela Alimentação Adequada e Saudável. Disponível em: https://alimentacaosaudavel.org.br/lei-que-prevemerenda-organica-nao-atinge-meta-em-sao-paulo/3564/. Acesso em 22 de jan. 2020. 
2. BARBOSA NVS, et al. Alimentação na escola e autonomia, desafios e possibilidades. Ciência \& Saúde Coletiva, 2013; 18: 937-945.

3. BATISTA MSA, et al. Ações do Programa Saúde na Escola e da alimentação escolar na prevenção do excesso de peso infantil: experiência no município de Itapevi, São Paulo, Brasil, 2014. Epidemiologia e Serviços de Saúde, 2014; 26: 569-578.

4. BEZERRA MA, et al. Saúde e nutrição em escolas públicas e privadas de Recife. Revista Brasileira de Saúde Materno Infantil, 2017; 17: 191-200.

5. BRASIL, 2009. In: Projeto de Lei nำ 4.910-B de 2009. Brasília: Câmara dos Deputados. Disponível em: https://www.camara.leg.br/proposicoesWeb/prop_mostrarintegra;jsessionid=299795B7B3FA3972596A0033CFA8A 8A6.node1 ?codteor=1039518\&filename=Avulso+-PL+4910/2009. Acesso em 23 de jan. 2020.

6. BRASIL, 2014. In: Planalto, Direito humano à alimentação adequada e soberania alimentar. Disponível em: http://www4.planalto.gov.br/consea/comunicacao/artigos/2014/direito-humano-a-alimentacao-adequada-esoberania-alimentar. Acesso em 23 de jan. 2020.

7. CAMOZZI ABQ, et al. Promoção da Alimentação Saudável na Escola: realidade ou utopia?. Caderno de Saúde Coletiva, 2015; 23: 32-37.

8. CASEMIRO JP, et al. Promover saúde na escola: reflexões a partir de uma revisão sobre saúde escolar na América Latina. Ciência \& Saúde Coletiva, 2014; 19: 829-840.

9. COELHO DEP e BÓGUS CM. Vivências de plantar e comer: a horta escolar como prática educativa, sob a perspectiva dos educadores. Saúde Soc, 2016; 3: 761-771.

10. CUNHA E, et al. A alimentação orgânica e as ações educativas na escola: diagnóstico para a educação em saúde e nutrição. Ciência \& Saúde coletiva, 2010; 15: 39-49.

11. DAVID CM, et al. Desafios contemporâneos da educação. 1nd ed. UNESP: São Paulo, 2015; $111 \mathrm{p}$.

12. FREITAS MCS, et al. Escola: lugar de estudar e de comer. Ciência \& Saúde Coletiva, 2013; 18: 979-985.

13. LIMA EE, SOUSA AA. Alimentos orgânicos na produção de refeições escolares: limites e possibilidades em uma escola pública em Florianópolis. Revista de Nutrição, 2011; 24(2): 263-273.

14. MANCUSO AMC, et al. O papel da alimentação escolar na formação dos hábitos alimentares. Revista Paulista de Pediatria, 2013; 31: 324-330.

15. MANCUSO AMC, et al. Educação Alimentar e Nutricional como prática de intervenção: reflexão e possibilidades de fortalecimento. Revista de Saúde Coletiva, 2016; 26: 225-249.

16. GLOBO. 2018. In: Os alunos tem direito a nutricionista nas escolas?. Rio de Janeiro. Disponível em: https://blogs.oglobo.globo.com/todos-pela-educacao/post/os-alunos-tem-direito-nutricionista-na-escola.html. Acesso em: 14 jan. 2020.

17. SANTOS F, et al. Avaliação da inserção de alimentos orgânicos provenientes da agricultura familiar na alimentação escolar, em municípios dos territórios rurais do Rio Grande do Sul, Brasil. Ciência \& Saúde Coletiva, 2014; 19: 14291436.

18. SÃO PAULO. 2015. In: LEIS MUNICIPAIS. São Paulo: Portal de divulgação da Prefeitura Municipal de São Paulo. Disponível em: https://leismunicipais.com.br/a/sp/s/sao-paulo/lei-ordinaria/2015/1614/16140/lei-ordinaria-n-161402015-dispoe-sobre-a-obrigatoriedade-de-inclusao-de-alimentos-organicos-ou-de-base-agroecologica-naalimentacao-escolar-no-ambito-do-sistema-municipal-de-ensino-de-sao-paulo-e-da-outras-providencias. Acesso em: 22 jan. 2020.

19. SILVA APF, SOUSA AA. Alimentos orgânicos da agricultura familiar no Programa Nacional de alimentação Escolar do Estado de Santa Catarina, Brasil. Revista de Nutrição, 2013; 26: 701-714.

20. SILVA EO, et al. Alimentação escolar e constituição de identidades dos escolares: da merenda para pobres ao direito à alimentação. Cadernos de Saúde Pública, 2018; 34: 16-22.

21. SOUSA AA, et al. Alimentos orgânicos e saúde humana: estudo sobre as controvérsias. Rev Panam Salud Publica, 2012; 31: 513-517.

22. TEO CRPA, et al. Direito Humano à alimentação adequada: Percepções e práticas de nutricionistas a partir do ambiente escolar. Trabalho, Educação e Saúde, 2017; 15: 245-267. 